\title{
Detection of Malignant Region of Diseased Images Using Thermal Camera
}

\section{Lakshman K ${ }^{1}$, Siddharth B Dabhade ${ }^{2 *}$, Sachin Deshmukh ${ }^{3}$, Ranjan Maheshwari $^{4}$ and Mrudul Behare ${ }^{1}$}

${ }^{1}$ NIELIT Aurangabad, Dr. B.A.M.U. CAMPUS, Aurangabad, MS, India

${ }^{2}$ MGM's, Dr. G.Y. Pathrikar College of Computer Science and IT, Aurangabad, MS, India

${ }^{3}$ Dr. Babasaheb Ambedkar Marathwada University, Aurangabad, MS, India

${ }^{4}$ Rajasthan Technical University Kota, RJ, India

*Corresponding Author: Siddharth B Dabhade, MGM's, Dr. G.Y. Pathrikar

College of Computer Science and IT, Aurangabad (MS), India.
Received: March 02, 2020

Published: July 01, 2020

(C) All rights are reserved by Siddharth B

Dabhade., et al.

\begin{abstract}
In medical science, abnormal growth of any cell is called cancer. Malignant region is nothing but the location of extra growth of any cell in the body. In this paper, we make attention on the different types of malignant region detection techniques using Thermal Infrared Images. We studied various imaging methods like thermal imaging, $x$-ray, magnetic resonance imaging (MRI), optical imaging of abnormal cell in human body. It is analyzed that malignant regions of body helps to prove the cancer cells those are present within the body. The early detection of malignant region helps in saving the life of an individual. To obtain thermal images, a thermal camera is used which can detect temperature variations in the body, as low as $0.1^{\circ} \mathrm{C}$. Thermal cameras capture the surface skin temperature which can vary due to emotion-specific, bio-physiological states in the human body.
\end{abstract}

Keywords: Medical Imaging; Malignant Region; Thermal Imaging; Cancer Biomedical

\section{Introduction}

Cancer is caused by an uncontrolled division of cells. Nowadays, cancer has spread in large amount in humans. Cancer is a multistage process in which normal cells will convert to abnormal growth, and which creates the malignant region with the tumors. World Health Organization (WHO) through its cancer research agency keeps record of classification of cancer causing agents through International Agency for Research on Cancer (IARC). Ageing is one more basic thing for the growth of cancer. The rate of cancer increases considerably with age, mostly due to a build-up of risks for specific cancers that increase with age. The in general threat increase is joined with the inclination for cellular restore mechanisms to be less efficient as a person grows older. The reasons of the cancer's mostly distant from inherent factors it can also contains 3 external causes like physical, chemical, and biological carcinogens. Smoke, tobacco, asbestos, food and water pollution due to definite chemical workings is called as chemical carcinogens and infections from viruses, parasites or bacteria are biological carcinogens. Roughly $70 \%$ of demises from cancer happen in low and middle-income countries.

\section{System development}

\section{Binarization}

Binarization is the process of converting a pixel image to a binary image. A binary image consists of pixels that have exactly two colors, typically black and white. In the older days, binarization was used for sending faxes. These days it's still important for things like digitalizing text or segmentation. It generates a two-level (binary) image whose pixel values correspond to 0 s and $1 \mathrm{~s}$. It is mainly useful when all center pixels have higher intensity values than surrounding pixels. It is a pixel (or point) operation, meaning it is applied to each pixel independently. 


\section{Segmentation}

Image segmentation is the process of separation of parts of a digital image into multiple segments (sets of pixels, also known as image objects). The goal of segmentation is to simplify and/or change the illustration of an image into something more significant and easier to analyze. Separation of a large image into several parts is nothing but the segmentation and which makes further processing simpler. These several parts that are rejoined will cover the entire image. Segmentation also depends on various characteristics like color or texture in the image. Medical Image Segmentation is the process of automatic or semi-automatic detection of boundaries within a 2D or 3D image. Moreover, many different methods (X-ray, CT, MRI, microscopy, PET, SPECT, Endoscopy, OCT, and many more) are used to generate medical images

\section{Region of interest}

A region in an image is a group of connected pixels with similar properties. Regions are important for the understanding of an image because they may correspond to objects in a scene. Region based segmentation technique and algorithms for 3D image. Image segmentation is the process by which the original image is partitioned into some meaningful regions. It is the base of higher level processing: e.g. object detection.

\section{Edge detection}

Edge detection is an image processing technique for finding the boundaries of objects within images. It works by identifying discontinuities in intensity. It is used for image segmentation and data extraction in image processing, computer vision, and machine vision. In image processing, an edge can be defined as a set of adjacent pixel positions where an sudden change of intensity (gray or color) values occur. Edges represent boundaries between objects and background. In this paper, we have concentrated on breast cancer detection using thermograph, which is more efficient than other techniques like mammograms. Flowchart gives the system sequence or operations those are made after capturing the image through thermal camera.

\section{Imaging techniques}

X-ray imaging

The mammograms for any specific abnormality can be detected by radiologist expert. Biopsy and anxiety for the patient involved due to individual reason error. To overcome this problem, use of computer-aided detection (CAD) system has used which is also used to reduce the human factor involvement and to help the radiologist to find out the mammograms automatically.

\section{Magnetic resonance imaging}

The data of MRI is important but, it may affect on normal human body. MRI is an advanced technology which gives the rich information about human self tissue anatomy. Different techniques to detect the cancer cell using the MRI: i) Dynamic contrast enhances MRI ii) Proton magnet resonance spectroscopy. For detecting and clinical management of breast cancer used the dynamic contrast enhances method. Nowadays major health disorder is breast cancer. It can cause death when it is not treated According to statistics a total of 1,660,290 new cancer cases and 580,350 cancer deaths are projected to occur in the United States in 2013. About 234,580 cancers are detected as in Cancer Journal for Clinicians.

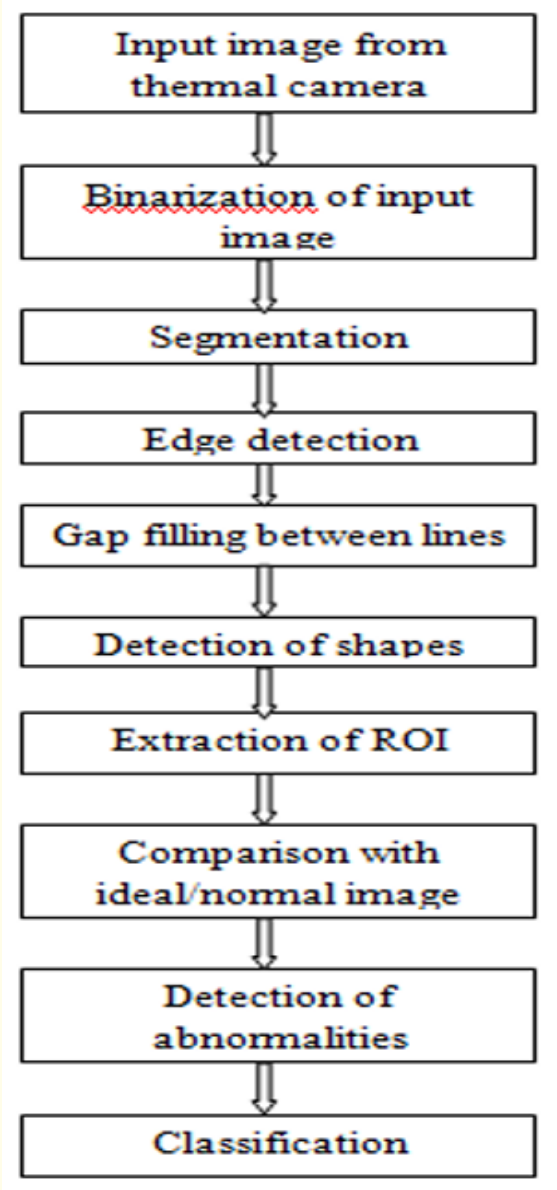

Figure 1: Flowchart of system.

The sensitivity of Mammography for breast cancer detection is moderate $(75 \%)$ and even reduced in women with dense breasts 
is $62 \%$. X- mammography has a $22 \%$ false positive rate in women under 50 . The mammography cannot identify whether the cancer is malignant tumors.

Drawbacks of MRI and ultrasonic:

- It is high cost,

- Low throughput,

- Low sensitivity.

Because of this drawback of MRI system, rarely used this system for the detection of the breast cancer. After this many techniques/methods comes out to detect breast cancer such as elastography, tomosynthesis, dedicated computer tomography (CT), positron emission tomography (PET), photo acoustic imaging and optical imaging.

\section{Mammography}

It is nothing but the technique of detection and diagnosis of tumors in the breasts using X-rays. Though it is similar to thermal imaging, it is unable to locate tumor in the dense breasts. If any younger lady gets a tumor then, it cannot be located or diagnosed by mammographic technique. False negative rate of mammograms is $40 \%$ for women ages from $40-49$, it means from 100 women having tumors, 40 mammograms miss the detection of breast cancer. Sometimes repetitive exposure to x-rays can cause cancer, so mammograms are needed to be replaced. Early detection of cancer, more sensitive to find more cancer, safer screening technology and it should be good for younger as well as older age women, these are the needs which should be fulfilled using some other technique. Mammogram screening is painful compared to thermal imaging, as compression of breast tissue is performed in mammography. Also higher number of exposure to x-ray can cause cancer to a healthy woman. Following images shows how mammograms are generated.

Hence, D.I.T.I. digital infrared thermal imaging technique is more efficient than the mammography. The temperature of normal breast tissue is lower than the pre-cancerous or cancer tissues' temperature. As a result, comparison can be performed on the basis of temperature value.

\section{Thermal imagining}

Thermal imaging technique is used in different medical operation to detect cancer. This gives the advanced advantage than the infrared imaging. The thermal imaging detects the different body

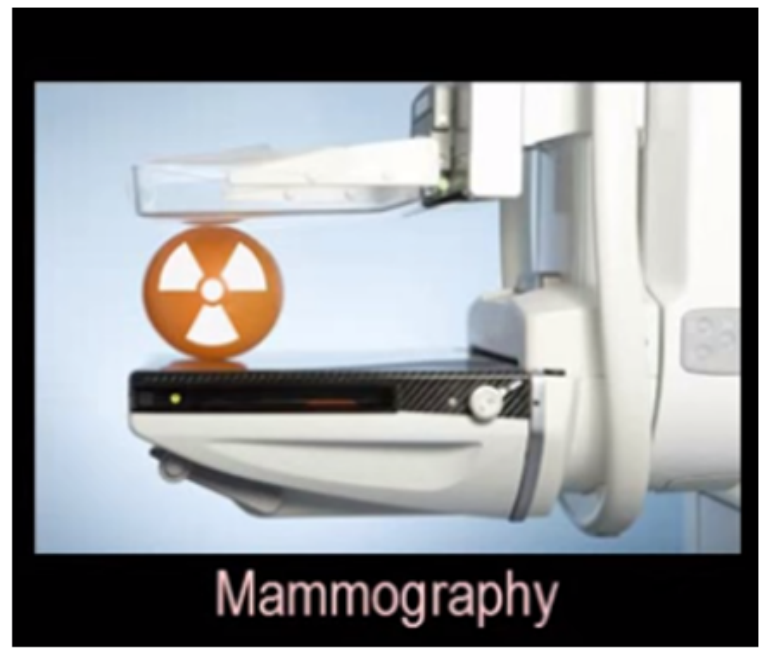

Figure 2: X-ray of breast tissue.

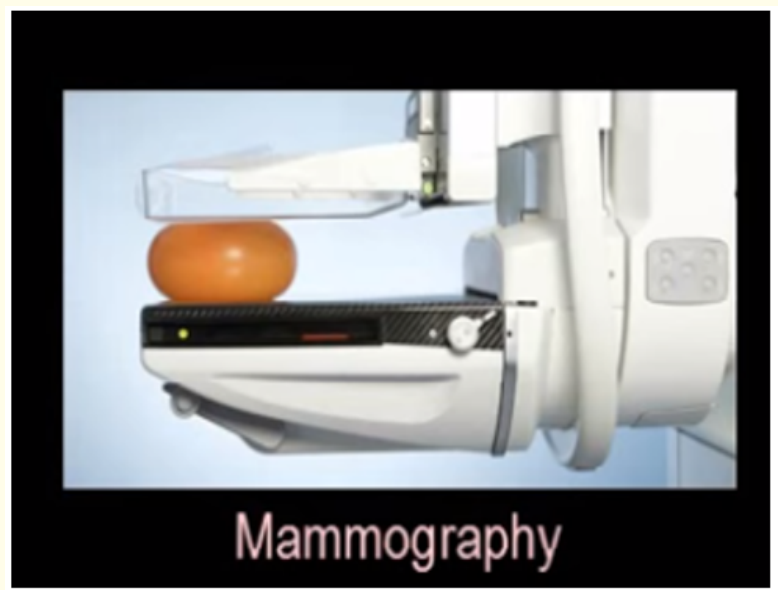

Figure 3: Breast tissue compression machine.

temp of human cell. According to this we are getting the information of the abnormal cell. If temperature changes according to that we get the abnormal growth of human body.

\section{Thermography camera}

We have used the infrared camera ThermaCAM S60 (FLIR Systems, International Main Office, Belgium) which has the following main features: thermal sensitivity 0.06 Cat30 C and 50/60 Hz, spectral range 7.5 - 13m (un-cooled micro bolometer with (320 

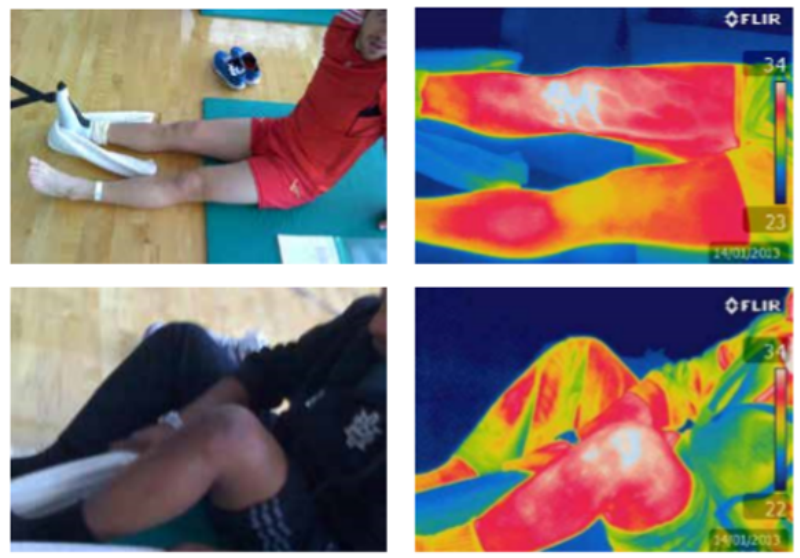

Figure 4: Thermal image showing inflammation of knee.

240 ) pixels). The camera be placed on a tripod in temperaturecontrolled room and calibrated after thermal stability is achieved (about 90 min after powering up).

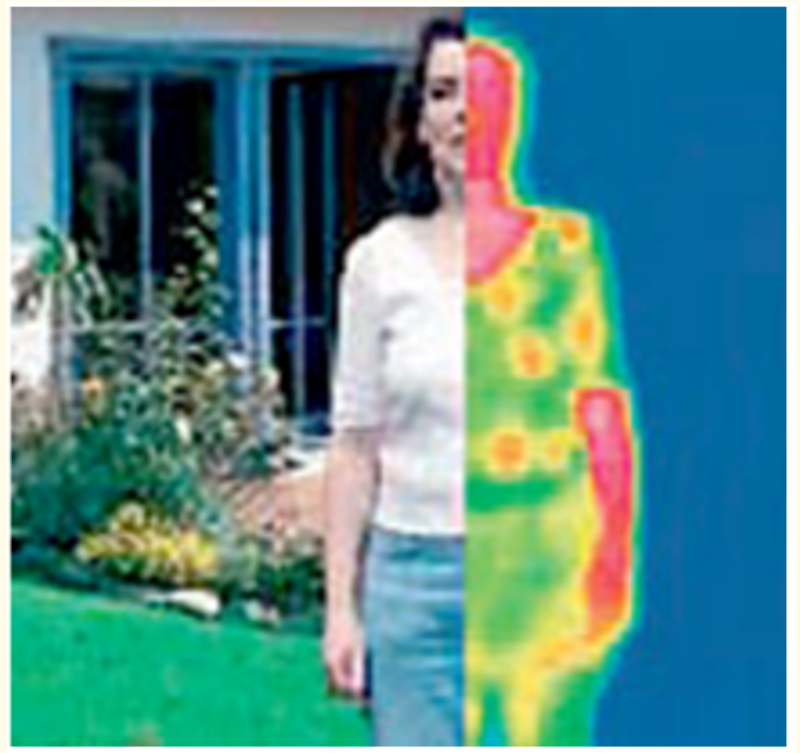

Figure 5: Image captured by thermography camera.

\section{Thermal image capturing process}

A special lens focuses the infrared light emitted by all of the objects in view. The detector components generate a very detailed temperature pattern called a thermogram.

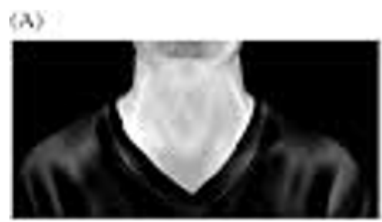

(C)
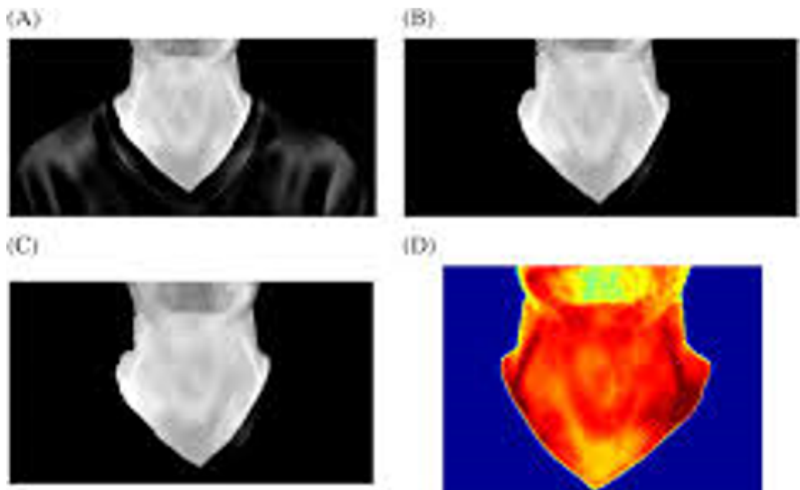

(D)

(E)

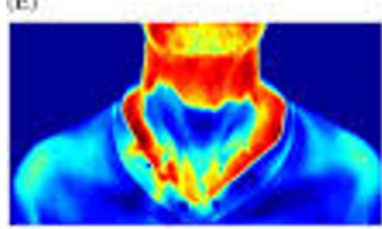

(F)
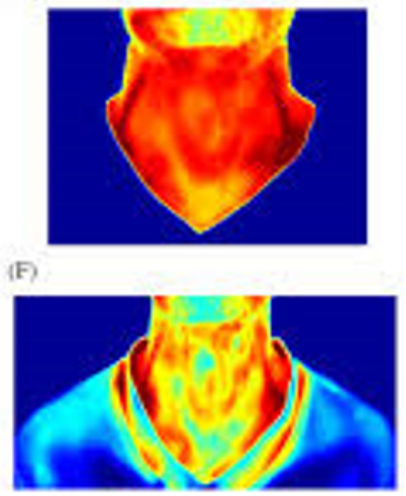

Figure 6: Analysis of thyroid thermographic images for detection of thyroid tumor.

\section{Breast thermograph}

Thermograph can be defined as a two dimensional function $\mathrm{g}(\mathrm{x}, \mathrm{y})$ where $\mathrm{x}, \mathrm{y}$ denotes the spatial co-ordinates and $\mathrm{g}(\mathrm{x}, \mathrm{y})$ denotes the vivacity at that pixel. Above thermographs are pseudo color thermographs in which both the colored red and white intensities equal up to the high temperature region and hence shows the presence of the abnormality. It is initiated accordingly thermograph of a regular person is symmetric and there is no unexpected variation in temperature. On the other hand, in abnormal thermographs there is an irregularity in temperature between the left and right breasts and in there is a sudden unexpected variation in temperature in the affected half. Nhan, Brian R., et al [14]. The asymmetry measured in terms of skewness, kurtosis, and absolute difference in mean, variance between the both halve. It is to create that there is important difference in Kurtosis and complete difference in mean in both the normal and abnormal thermographs [1-18].

The temperature of normal breast tissue is lower than the precancerous or cancer tissues' temperature. As a result, comparison can be performed on the basis of temperature value. Statistical analysis of detection of breast cancer shows that thermal imaging technique correctly detects $92 \%$ of the breast cancer cases. 

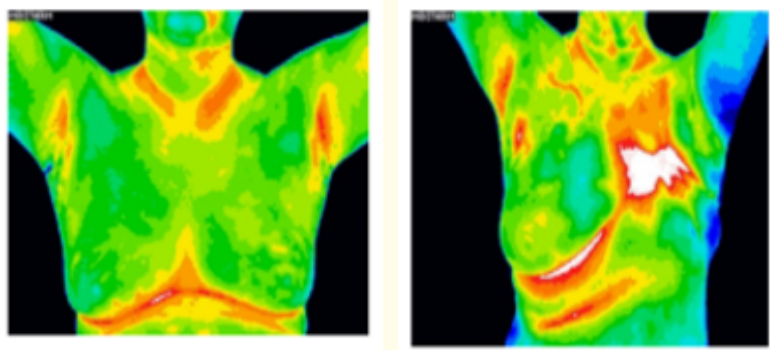

Figure 7: Normal and abnormal thermograph of breasts.

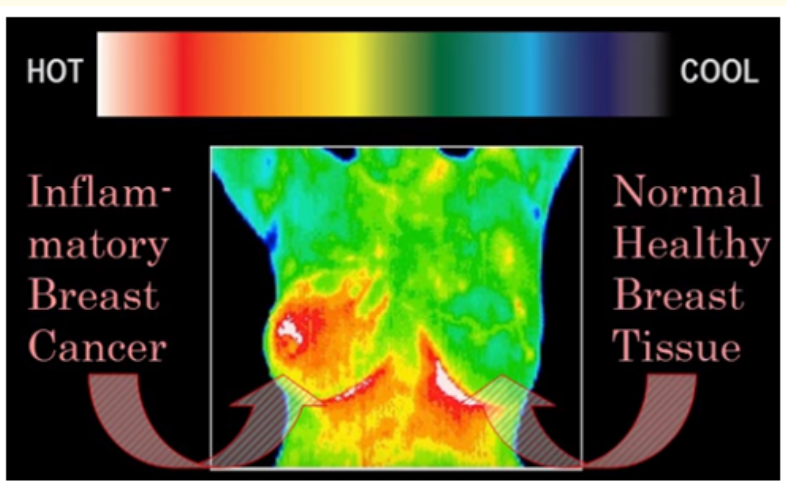

Figure 8: Comparison between normal and inflammatory tissue.

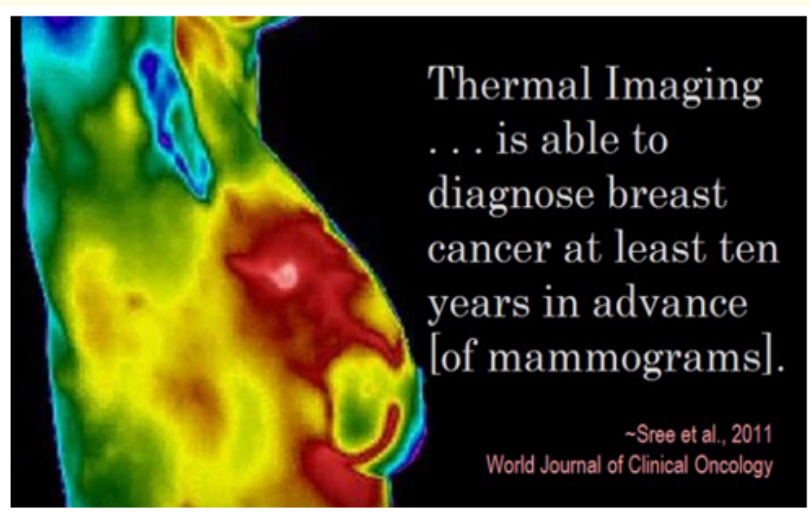

Figure 9: Early detection of breast cancer.

\section{Conclusion}

In this paper detection and classification of diseased parts of human body is performed using thermal camera images. Compari- son of the features of normal images with the diseased one are calculated and based on the results classification is made. This paper mainly works on early detection of breast cancer, as it is the most common type of cancer these days and also difficult to find early using other techniques like mammograms. We analyzed and research on all the techniques to find cancer cell/abnormal growth cell in human body. Thermal image processing is emerging technique to find out the cancer cell in human body. In order to understand this possible, the detailed physical mechanisms of TIR emittance from human anatomy must be elucidated. Medical TIRI must also experience a thorough holistic systems analysis, from which objective clinical protocols and contextual best practices may be established.

\section{Bibliography}

1. G Eason., et al. "On Certain Integrals of Lipschitz-Hankel Type Involving Products of Bessel Functions". Philosophical Transactions of the Royal Society A London A247 (1955): 529-551.

2. J Clerk Maxwell. "A Treatise on Electricity and Magnetism, $3^{\text {rd }}$ Edition". Oxford: Clarendon 2 (1892): 68-73.

3. IS Jacobs and CP Bean. "Fine Particles, Thin Films and Exchange Anisotropy". In Magnetism, GT Rado and H. Suhl, Eds. New York: Academic 3 (1963): 271-350.

4. K Elissa. "Title of Paper If Known”. Unpublished.

5. R Nicole. "Title of Paper With only First Word Capitalized". J. Name Stand. Abbrev., In Press.

6. Y Yorozu., et al. "Electron Spectroscopy Studies on MagnetoOptical Media and Plastic Substrate Interface". IEEE Transl. J. Magn. Japan 2: 740-741, August 1987 Digests 9th Annual Conf. Magnetics Japan, P. 301 (1982).

7. M Young. "The Technical Writer's Handbook". Mill Valley, CA: University Science (1989).

8. J Rumiński., et al. "Thermal Parametric Imaging In The Evaluation of Skin Burn Depth". IEEE Transactions on Biomedical Engineering 54 (2007): 303-312.

9. BF Jones. "A Reappraisal of The Use of Infrared Thermal Image Analysis In Medicine". IEEE Transactions on Medical Imaging 17 (1998): 1019-1027.

10. SA., et al. "Infrared Thermography and Image Analysis For BioMedical Use". Periodicum Biologorum 113 (2011): 385-392. 
11. VVoronin., et al. "Thermal Image Enhancement Algorithm Using Local and Global Logarithmic Transform Histogram Matching With Spatial Equalization". Proceeding IEEE Southwest Symp. Image Anal. Inter- Pret (2018): 5-8.

12. Y Cho. "Automated Mental Stress Recognition Through Mobile Thermal Imaging". 2017 7th Int. Conf. Affect. Comput. Intell. Interact. ACII 2017, 2018-Janua (2018): 596-600.

13. U Snekhalatha., et al. "Thermal Image Analysis and Segmentation of Hand In Evaluation of Rheumatoid Arthritis". 2012 International conference on computer, communication and informatics, ICCCI (2012).

14. BR Nhan and T Chau. "Classifying Affective States Using Thermal Infrared Imaging of The Human Face". IEEE Transactions on Biomedical Engineering 57 (2010): 979-987.

15. S Mukhopadhyay., et al. "A New Paradigm of Oral Cancer Detection Using Digital Infrared Thermal Imaging". 9785 (2016): 97853I.

16. H Yang., et al. "Imaging and Its Preliminary of Breast Disease Assessment". IEEE Complex Med Eng (2007): 1071-1074.

17. S Sruthi and M Sasikala. "A Low Cost Thermal Imaging System For Medical Diagnostic Applications". 2015 Int. Conf. Smart Technol. Manag. Comput. Commun. Control. Energy Mater (2015): 621-623

18. Https://Www.Youtube.Com/Watch?V=Txpareputme

\section{Assets from publication with us}

- Prompt Acknowledgement after receiving the article

- Thorough Double blinded peer review

- Rapid Publication

- Issue of Publication Certificate

- High visibility of your Published work

Website: www.actascientific.com/

Submit Article: www.actascientific.com/submission.php Email us: editor@actascientific.com

Contact us: +919182824667 